\title{
Research Progress on Ecological Protection and Restoration of Urban Wetlands in China
}

\author{
Wen Zhan ${ }^{1}$, Shouyun Shen ${ }^{1}$, Huifeng Cheng ${ }^{2}$ \\ ${ }^{1}$ College of Landscape Architecture, Central South University of Forestry and Technology, \\ Changsha Hunan, 410004, China \\ ${ }^{2}$ Changsha Environmental Protection College, Changsha Hunan, 410000, China
}

Key words: Urban wetland, Protection and restoration, Research progress.

\begin{abstract}
As urbanization course speeds up in China, urban wetlands are faced with a series of problems such as serious pollution, sharp decrease of area, eutrophication aggravation, acceleration of biotic intrusion, lack of management system and weak protection awareness. Ecological protection and restoration of urban wetlands become the research hotspots of city and wetland topics. This paper discusses the measures about ecological protection of urban wetlands in China and research progress on ecological restoration technologies, combines current situations of urban wetlands in China to propose countermeasures and suggestions on ecological protection and restoration of urban wetlands and provides beneficial reference for promoting virtuous circle of urban wetland ecosystem and driving comprehensive development of urban economic benefit and ecological benefit.
\end{abstract}

\section{Introduction}

The wetland refers to artificial or natural and temporary or long-term marshland, flowing or still brackish water, fresh water, salt water, water area or peatland as well as the water area less than $6 \mathrm{~m}$ in the low tide period. This is the definition of wetland in International Wetland Convention[1]. The definition of urban wetland has not been generally accepted. According to relevant research results, it is believed in this paper that, urban wetland refers to the ecosystem with water area and land area transition in the urban region such as estuary, coast, shallow lake and marsh, artificial and natural pond, paddy field, sewage treatment plant and water conservation district. The urban wetland is in the city and plays the role in adjusting urban ecological environment and serving the society. To be specific, the urban wetland plays a decisive role in urban resource supply, disaster prevention and control, environment adjustment, environment beautifying, and social culture support. Thus, urban wetland protection and restoration should be further valued during scientific and rational development of cities so as to facilitate normal exertion of urban wetland functions and to promote sound development of urban ecosystem and social economy.

In recent years, urban wetland destruction and pollution phenomena have become increasingly serious, as urbanization course speeds up and urban population is on the increase. The phenomena that urban wetlands are transformed into urban land and that industrial wastewater and residential sewage are discharged into urban wetlands also have become more and more frequent. Urban wetlands present the problems of fragmentation aggravation, uneven distribution, the decrease of area and severe water pollution etc. ecological benefit and social benefit of urban wetlands are gradually cut down, and the adverse factors are fed back to urban residents. Thus, ecological protection and restoration of urban wetlands are pressing. In the early 19th century, foreign relevant researchers started to research protection and restoration of urban wetlands. In 2003, China issued Nationwide Wetland Protection Project Planning which covers ideological guidance for wetland protection, task objective setting, key construction and planning as well as construction measure planning etc.[2] 
Relevant researches and policies provide beneficial theoretical suggestions and system safeguard for urban wetland protection and restoration in China. To further promote the effect of urban wetland protection and restoration, this paper deeply analyzes main problems of urban wetlands, research progress of urban wetland protection and restoration and suggestions on urban wetland protection and restoration.

\section{Main problems of urban wetlands}

The urban wetland as urban ecological infrastructure is faced with various hazards and even destructiveness problem under the trend of urbanization. The main problems include sharp decrease of area, serious wetland pollution, eutrophication aggravation, biotic intrusion aggravation, weak wetland management system and weak protection awareness. All these problems continuously damage urban wetland ecosystem and functions.

\section{Sharp decrease of area}

The sharp decrease of urban wetland area is a global issue. In the urbanization course, urban wetland protection and restoration measures are incomplete. Wetland development is improper, and wetlands are occupied by urban building land in quantity. Thus, urban wetland area cuts down sharply. Relevant researches show that urban wetland area in America dropped sharply from $69000 \mathrm{~km} 2$ to below a half in the period from the early 20th century to 1990, and that excessive land utilization made American urban wetland area cut down sharply[3]. In China, urban wetland area also has presented the sharp decline trend in recent 40 years. Coastal wetland has decreased by $21900 \mathrm{~km} 2$, amounting to a half of original coastal wetland area. The wetland in Beijing has cut down to 0.33km2 since the middle 20 century. Nowadays, the decline trend of global urban wetland area still continues. As urban wetland area decreases and urban heat island effect intensifies, urban wetland ecosystem has suffered serious damages.

\section{Aggravation of wetland pollution and eutrophication}

Immoderate discharge of domestic wastewater and industrial wastewater into wetlands results in the aggravation of urban wetland pollution and eutrophication. Rapid economic development promotes industrial development, and human living standards also improve continuously. However, environmental protection awareness, environmental protection provisions and measures fail to keep up with the times. Domestic and industrial wastewater, poultry pollutants and chemical fertilizer pollutants are discharged into urban wetlands without treatment in quantity, which surpasses the self-cleaning capacity of wetlands. Thus, water quality of wetlands drops seriously. Meanwhile, wetland water eutrophicates, and wetland environment is seriously damaged. Wetland pollution and eutrophication cause massive death of biology in wetlands. Thus, the number of biological species in wetlands decreases sharply, and wetland environment and surroundings suffer severe damages.

\section{Biotic intrusion aggravation}

With irrational urban wetland governance, China blindly introduces non-indigenous organisms, which happens continuously. Biotic intrusion of urban wetlands results in the extinction of some species, and imbalanced wetland ecological environment in Chinese urban wetlands. In addition, both the yield and quality of agriculture, forestry, fishery and animal husbandry suffer severe impacts. Economic loss and governance cost are enormous. The data show that biotic intrusion of American urban wetlands leads to the economic loss of 3.8 billion annually. Spartina anglica which was introduced by China in 1960s and used to create land with silt, protect sand and strengthen dykes caused ecological disaster in most watercourses of south China. From 1980s, water hyacinth was introduced in many urban wetlands of south China, which gave rise to disasters caused by flooding water of lakes and watercourses and brought disasters to more than 10 provinces and cities. At present, biotic intrusion trend of urban wetlands still presents the acceleration state in China, which forms a great threat to social and economic development as ell as ecological balance. 


\section{Weak wetland management system and protection awareness}

Urban wetland management system construction starts late in China. Besides, it is hard to coordinate due to a wide range of management, development and utilization and wide area. As well, human wetland protection awareness is weak, which aggravates urban wetland damages. So far, China has failed to issue uniform laws and regulations for urban wetland protection. Meanwhile, the breadth, depth and force of wetland protection publicity and education are insufficient. The contradiction between economic benefit and ecological environmental protection is sharp in urban wetland management. The whole society is generally short of urban wetland protection awareness. There is lack of the cognition for urban wetland value and importance.

\section{Research progress of urban wetland protection and restoration}

\section{Principle and objective research progress}

Urban wetland protection and restoration should aim at balance and development of economic benefit and ecological benefit and follow corresponding principles and objectives. Based on current researches, three objectives and six principles about urban wetland protection and restoration are summarized in this paper. The three objectives are as follows: (1) ecological objective: to increase biological diversity of urban wetlands and restore biocenosis balance of wetlands; (2) landscape objective: to improve aesthetic value of urban wetlands; (3) water quality objective: to improve water quality of urban wetlands and restore the hydrological environment. The six principles include the following: (1) the principle of maximum benefit and minimum risk: maximization of economic benefit and ecological benefit, and minimization of hazards and risks; (2) priority principle: preferential protection and restoration of urban wetlands which suffer severe damages; (3) feasibility principle: feasibility of urban wetland protection and restoration plan, implementation and technology; (4) local principle: local culture and traditional culture protection in the process of urban wetland protection and restoration; (5) principle of continuity and integrity: guarantee rational connection of urban wetlands in process of urban wetland protection and restoration and guarantee continuity and integrity of wetland system; (6) aesthetic principle: make sure urban wetlands follow the principle of green and health and reflect peculiarity, cleanness, ornamental value and joviality of wetlands.

\section{Research progress on ecological protection measures of urban wetland}

The researches on urban wetland protection measures mainly cover the following aspects: building ecological preservation area of wetlands, ecological tourism, enhancing wetland legislation construction and building wetland parks. Chilika Lake in India achieved effective wetland protection and utilization through sustainable management, community participation promotion, close monitoring and estimation of wetland environment quality. America effectively relieved the bad situation of urban wetlands through establishing wetland relief banks and gave urban wetland protection fund compensation, which promotes the innovation of urban wetland protection system. Mai Po Wetlands Protection Zone in Hong Kong reached the objective of wetland protection and utilization through carrying out ecological tourism and ecological education, formulating and implementing feasible wetland protection rules and plans, and conducting sustainability management[5].

Many experts in China have researches ecological protection of urban wetlands. Zhou Lingxia studied development and utilization status of urban wetland in Pukou District, Nanjing City and considered that comprehensive treatment of water and wetland water source supply are important measures for urban wetland protection. On the other hand, establishing wetland ecological preservation zones and wetland parks also contributes to the protection of urban wetlands. In the opinion of Li Haisheng, enhancing construction and management of natural conservation area and strengthening wetland park construction and management are important measures to protect urban wetlands in Shenzhen. Ren Liyan et al researched the influence of Hangzhou Bay city planning and 
industrial development on wetland protection and indicated that wetland protection should be enhanced through adjusting land use planning, wetland occupancy compensation and urban wetland park building etc.

\section{Rsearch progress on urban wetland restoration technology}

Urban wetland restoration requires engineering or non-engineering measures as well as relevant restoration techniques to restore or rebuild wetland structure, functions and characteristics so as to make wetlands restore self-maintenance state and ecological balance. Currently, urban wetland restoration technologies mainly include biological measures, physical measures, and chemical measures. Biological measures promote urban wetland restoration through ecological bank protection, biomanipulation, artificial aeration and aquatic vegetation restoration. Physical measures promote urban wetland restoration through dyke engineering, water and soil engineering, bottom mud sulfation, water dilution, isolation and coverage (masking method). Chemical measures promote urban wetland restoration through adding chemical drugs and microorganism and building artificial wetlands. Domestic and oversea application cases of urban wetland restoration technologies are as follows:

America gained good effects of environmental improvement of urban wetlands through such measures as sewage discharge control, restoration plan, and restoration effect evaluation in 1975-1985. Japan Lake Kasumigaura wetland restoration plan effectively prevented eutrophication of urban wetland water through domestic sewage treatment system reform, sewage dephosphorization measures and resource recovery system, and effectively facilitated water quality improvement and ecological environment balance of urban wetlands through purification system, beneficial microorganism and bottom mud dredging. In China's Agenda in the 21st Century formulated in 1994, wetland ecosystem protection \& restoration and water pollution control are deemed as the long-term goal of wetland protection and restoration. This provides powerful system guarantee for urban wetland protection and restoration in China. Besides, eutrophication situation of urban wetlands such as East Lake, Taihu Lake and Chaohu Lake have been relieved effectively[6]. Furthermore, from 1970s, China deeply studied ecological restoration and reconstruction of degraded wetlands, and the researches mainly focus on degraded mangrove forest restoration and comprehensive utilization.

\section{Suggestions on urban wetland protection and restoration}

Under the background of economic boom, urban wetland protection and restoration measures must keep pace with the urbanization and continuously seek innovation and development in the balance of economic benefit and ecological benefit. In accordance with specific national conditions of China, the following aspects should be enhanced in urban wetland protection and restoration: (1) urban wetland protection and restoration should be brought into legal system. China belongs to a state with legal system. The application of legal weapon in urban wetland protection and restoration is more authoritative and operable. (2) The education and publicity of urban wetland protection and restoration should be enhanced. The idea dominates human behaviors. Thus, it is necessary to deepen people's identity sense for urban wetland protection and restoration through knowledge education and law popularization. (3) Ecosystem monitoring and evaluation should be strengthened, and protection and restoration should be carried out in real time. Effective monitoring and evaluation can nip the dangers in the bud. Dynamic monitoring of urban wetlands with modern 3S technology contributes to scientific decision making and implementation of urban wetland protection and restoration. (4) Ecological tourism of urban wetlands should be developed greatly to promote economic development of urban wetland district, which can effectively relieve the conflict between economic benefit and ecological benefit, and facilitate the course of urban wetland protection and restoration. (5) The research and innovation of urban wetland protection and restoration technology should be enhanced. Meanwhile, international cooperation should be strengthened. Technical 
innovation and international cooperation for urban wetland protection and restoration can effectively improve the level and efficiency of urban wetland protection and restoration.

\section{Conclusion}

The researches on urban wetland protection and restoration in China should focus on the balance between economic benefit and ecological benefit urban wetland district. The implementation of specific protection and restoration measures and technologies should be based on the actual conditions. Under the condition of effective protection and restoration of urban wetlands, great development of economy in the urban wetland district is the core of urban wetland district development.

\section{Acknowledgement}

Fund program: excellent youth project of Education Department of Hunan Province (14B196).

\section{References}

[1] Wu Fang, On Research Progress of Domestic and Oversea Urban Wetland Protection Planning, Xiandai Horticulture, 2016, 1(03): 65-66.

[2] Zhang Bo, Restoration, Utilization and Sustainable Development of Urban Wetlands - Protection and Construction of Phase I and Phase II of Guangdong Haizhu National Wetland Park, Xiandai Horticulture, 2015, 1(02): 49-50.

[3] Wang Yang, Study on Urban Wetland Park Planning and Design under Background of Sustainable Development - Case Study of Wenzhou Sanyang Wetland, Chinese Horticulture Abstracts, 2017, 3(01): 119-120.

[4] Liu Xingxing, Shen Shouyun, Preliminary Study on Ecological Planning and Design of Urban Wetland Park_— Taking the Overall Planning Concept of Wuzhou Canghai Wetland Parkas for Example, Journal of Green Science and Technology, 2017, 1(03): 6-8.

[5] Zhao Songying, Qin Jiaqi, Research on Ecosystem Health Evaluation of Changchun Beihu Wetland Based on PSR Model, Industrial Safety and Environmental Protection, 2017, 43(03): 71-72.

[6] Wag Qian, Wetland Park Design Based on Sponge City Concept - Case Study of Harbin Qunli Yuhong Park, China Flowers \& Horticulture, 2017, 2(01): 54-55. 